\title{
EAS primary particle parameter estimation with the complex Pamir-XXI detector array
}

\author{
V.I. Galkin ${ }^{1,2, a}$, A.S. Borisov ${ }^{3, b}$, R. Bakhromzod ${ }^{1,4}$, V.V. Batraev ${ }^{1}$, S. Latipova ${ }^{5}$, and A. Muqumov ${ }^{1,4}$ \\ ${ }^{1}$ Lomonosov Moscow State University, Faculty of Physics, Moscow, Russia \\ ${ }^{2}$ Lomonosov Moscow State University, Skobeltsyn's Institute of Nuclear Physics, Moscow, Russia \\ ${ }^{3}$ Lebedev Physical Institute of the Russian Academy of Sciences, Moscow, Russia \\ ${ }^{4}$ Umarov Physical-Technical Institute of Academy of Sciences of Tajikistan, Dushanbe, Tajikistan \\ 5 Tajik National University, Faculty of Physics, Dushanbe, Tajikistan
}

\begin{abstract}
Some new developments in EAS methods made in the framework of the Pamir-XXI project are presented. First, primary energy and direction definition accuracies by a network of fast scintillators are considered, optimum network cell size is defined for $10 \mathrm{PeV}$ showers. Second, the same accuracies for a network of fast optical (Cherenkov) detectors are considered for $30 \mathrm{TeV}-10 \mathrm{PeV}$ showers. Third, the possibilities of separation of EAS initiated by protons, nitrogen and iron nuclei of 1 and $10 \mathrm{PeV}$ energies using a wide-angle Cherenkov telescope are discussed. Finally, the results of the extraction of 30-50 TeV gamma showers from the proton shower background with the same telescope are presented.

Presumably, our developments can help in the study of PCR mass composition and ultra high energy gamma ray astronomy in other projects.
\end{abstract}

\section{Introduction}

The Pamir-XXI project was originally planned as a multi-component study of EAS in a wide energy range $30 \mathrm{TeV}-1 \mathrm{EeV}$ bearing in mind the solution of astrophysical problems (detailed studies of PCR energy spectrum and mass composition, detection of $\gamma$-rays of point-like and diffuse sources above $30 \mathrm{TeV}$ ) and the problems of hadron interaction at super high energies including exotic phenomena in EAS cores.

At present there is no certainty in the terms of realizing the project, but we still continue developing the experimental procedures using detailed simulations of EAS characteristics. To reach the denoted aims a complex detector array is required, incorporating numerous detectors of different types [1].

In this paper we concentrate on the analysis and selection of optimal detector configurations for:

- Cherenkov $\gamma$-ray astronomy;

- PCR energy spectrum and mass composition studies.

\section{Statement of the problem and the solution logic}

In order to succeed in the above mentioned studies it is enough to measure primary particle energy $E_{0}$, type (mass), arrival direction $\vec{\theta}$ and core location $\vec{x}_{\text {core }}$ at the observation level. These parameters are usually considered to be equally demanding with regard to the

\footnotetext{
a e-mail: v_i_galkin@mail.ru

b e-mail: asborisov55@mail.ru
}

amount of experimental data used while making the parameter estimates. Our analysis shows this is not the case as the primary type (mass) proves to be much more demanding.

That is why we insist that a detector array and data processing methods should be optimized with respect to the informatively most exacting problems, namely, the primary mass estimation and the $\gamma$-event selection.

For the measurements to be as consistent as possible one must consider the most information-rich component to be the reference component for all other measurables. Among the EAS components the Cherenkov light (CL) is definitely the most informative and makes it possible to solve all stated problems within the energy range mentioned even with a small duty cycle $(\leq 10 \%)$.

Taking into account these statements, one is forced, first of all, to construct an array of Cherenkov detectors optimized for the solution of primary type/mass estimation problem. The array should analyze both Cherenkov pulses (spatial-temporal distribution of CL) and Cherenkov [angular] images (CL spatial-angular distribution) and thus will include a network of widely spaced fast optical detectors and a set of a few optical telescopes.

A network of charged particle detectors will be used at $E_{0}>1 \mathrm{PeV}$ for evaluation of $E_{0}, \vec{\theta}, \vec{x}_{\text {core }}$ to help the central calorimeter and muon detectors (presumably trackers) to estimate the primary mass.

The method for the primary mass estimation by charged particle data (when finally established) can be tuned with the help of the respective Cherenkov light method. 


\section{Artificial events}

\subsection{Generation}

Artificial events for the observation level $4250 \mathrm{~m}$ a.s.l. were generated with CORSIKA6.990/QGSJET01 [2] for a set of fixed $E_{0}$ from $30 \mathrm{TeV}$ to $100 \mathrm{PeV}$ and different primary types: $\mathrm{p}, \mathrm{He}, \mathrm{N}, \mathrm{S}, \mathrm{Fe}, \gamma$. CL spatial-temporal and spatial-angular distributions and CORSIKA standard particle files were stored.

As for super high energy hadron interaction model used, one might object that QGSJET01 is not the one that best fits the EAS data pool. Our answer is: nobody knows what is the true hadron interaction model, although all the models incorporated in CORSIKA reproduce EAS development satisfactorily and definitely recreate relative changes of the EAS characteristics with $E_{0}$ and $A$ (primary mass). This is what matters most of all when one looks for a function of measurable values that can help to distribute EAS events by A. When such a function is found one can correct it for the hadron interaction model and even do without any model using experimental distributions.

\subsection{Processing}

To process the spatial-temporal data of simulated events simple polynomial models were used for CL and particle lateral distributions $\mathrm{LD}(\mathrm{R})$ and fronts $\tau(\mathrm{R})$ :

$$
\begin{aligned}
\mathrm{LD}(\mathrm{R}) & =\mathrm{a}_{0} /\left(1+\mathrm{a}_{1} \mathrm{R}+\mathrm{a}_{2} R^{2}+\mathrm{a}_{3} R^{3}\right), \\
\tau(R) & =R\left(a_{0}+a_{1} R+a_{2} R^{2}\right) .
\end{aligned}
$$

The shower direction definition is done simultaneously with the core location search by fitting the measured front arrival times by the front model and the measured number of photons (photoelectrons)/particles by the lateral distribution model. During this fit $\tau(\mathrm{R})$ and $\mathrm{LD}(\mathrm{R})$ parameters are also varied so that we obtain the best front and lateral distribution approximations for each event. $\mathrm{LD}(\mathrm{R})$ is further integrated within a circle of a certain radius to form an estimate of $E_{0}$.

Processing of CL angular images does not require any model functions, it comprises the location of a spot's long axis and integration over certain regions of field of view (FOV). The primary direction estimate by spatial-temporal data is used.

\section{Optical part of Pamir-XXI detector array}

The optical part of the detector array uses spatial-temporal and spatial-angular distributions of CL for the estimation of primary particle parameters and, thus, must incorporate two sets of detectors (Fig. 1).

\subsection{Multiple use of event Cherenkov data}

Event sample volumes vary from 200 for $30-100 \mathrm{TeV}$ primaries to 30 for $100 \mathrm{PeV}$ primaries but CL data of each event includes:

- a $600 \times 600 \times 302$ histogram of spatial-temporal distribution covering a $600 \mathrm{~m} \times 600 \mathrm{~m}$ carpet and $\sim 300$ ns delay interval and
Pamir-XXI optical

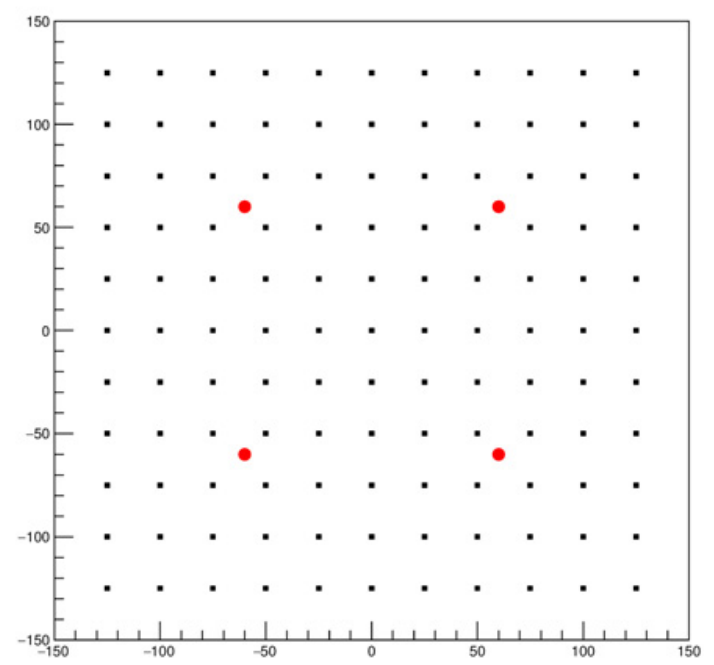

Figure 1. Optical detector array layout used in simulations: 1) rectangular network of $11 \times 11$ wide angle fast optical detectors of area $\sim 1 \mathrm{~m}^{2}$ and aperture $\sim 1$ sr set with $25 \mathrm{~m}$ step (squares), which must be able to determine the shower direction with an accuracy of at least $0.1^{\circ}$, core location with an accuracy of $\sim 1 \mathrm{~m}$ and the primary energy within $15 \%$ accuracy and also makes it possible to analyse the CL pulse shape; 2) optical image telescopes (circles) with mirrors of area $\sim 4 \mathrm{~m}^{2}$, field of view diameter of about $30^{\circ}$ and pixel diameter of $\sim 0.8^{\circ}$ spaced by $\sim 100 \mathrm{~m}$, which should enable the analysis of the CL angular distribution close to the shower core and be sensitive to the primary particle mass.

\section{- a $250 \times 250 \times 108 \times 108$ histogram of spatial- angular distribution within a $500 \mathrm{~m} \times 500 \mathrm{~m}$ square of observation level with every $2 \mathrm{~m} \times 2 \mathrm{~m}$ bin imitating an imaging telescope of $27^{\circ} \times 27^{\circ}$ field of view.}

Thus, each event can be used many times by choosing different $1 \mathrm{~m} \times 1 \mathrm{~m}$ bins of the 1 st histogram as detectors of fast network and different $2 \mathrm{~m} \times 2 \mathrm{~m}$ bins of the $2 \mathrm{nd}$ histogram as imaging telescopes.

\subsection{CL spatial-temporal distribution processing results}

Uncertainties of core location and primary direction determination by fast optical detector network meet the above set requirements $\left(\sim 1 \mathrm{~m}\right.$ and $\left.<0.1^{\circ}\right)$. The results for pure $\mathrm{CL}$ signal in photons and signal + night sky background (BG) $\left(10^{8} \mathrm{~cm}^{-2} \mathrm{sr}^{-1} \mathrm{~s}^{-1}\right)$ in photoelectrons are almost the same for $E_{0} \geq 1 \mathrm{PeV}$. The situation changes for primary energies $30-100 \mathrm{TeV}$ but the uncertainties still lie within the pre-set limits which gives hope to detect gamma-events in this energy range (Table 1).

The primary energy $E_{0}$ is estimated as an integral CL300 of CL lateral distribution (LD) over a circle of radius $300 \mathrm{~m}$. Parameters of $\mathrm{LD}$ are obtained as a result of a joint fit of CL LD and front models to CL spatialtemporal data of individual EAS. Uncertainties of CL300 for $E_{0} \geq 1 \mathrm{PeV}$ are indifferent to night sky $\mathrm{BG}$ and conversion to photoelectrons (Table 2). The situation is different for the 30-100 $\mathrm{TeV}$ range: mixing of CL and $\mathrm{BG}$ photons and their conversion to photoelectrons increase the uncertainties (Table 3). Introduction of a threshold 
Table 1. Core location, $m$, and primary direction, ${ }^{\circ}$, uncertainties.

\begin{tabular}{lllll}
\hline event/signal & core loc.mean & core loc.RMS & prim.dir.mean & prim.dir.RMS \\
\hline $60 \mathrm{TeV}$ p, CL & 1.6 & 0.93 & 0.044 & 0.033 \\
$60 \mathrm{TeV}$ p, CL+BG & 2.6 & 1.9 & 0.076 & 0.064 \\
$60 \mathrm{TeV}$ p, CL+BG, $>$ 200phel & 2.4 & 1.6 & 0.059 & 0.048 \\
\hline $30 \mathrm{TeV} \gamma, \mathrm{CL}$ & 1.0 & 0.59 & 0.030 & 0.020 \\
$30 \mathrm{TeV} \gamma, \mathrm{CL}+\mathrm{BG}$ & 1.8 & 1.3 & 0.058 & 0.052 \\
$30 \mathrm{TeV} \gamma, \mathrm{CL}+\mathrm{BG},>$ 200phel & 1.5 & 1.1 & 0.051 & 0.046 \\
\hline
\end{tabular}

Table 2. CL300 relative fluctuations for $E_{0} \geq 1 \mathrm{PeV}, \%$.

\begin{tabular}{lccc}
\hline$E_{0}, \mathrm{PeV}$ & proton & nitrogen nucleus & iron nucleus \\
\hline 1 & 15 & 10 & 8 \\
10 & 14 & 8 & 5 \\
\hline
\end{tabular}

Table 3. CL300 relative fluctuations for $E_{0}=30-100 \mathrm{TeV}, \%$.

\begin{tabular}{lccc}
\hline $\begin{array}{l}E_{0}, \mathrm{TeV}, \\
\text { prim.patricle }\end{array}$ & $\begin{array}{c}\mathrm{CL} \\
\text { photons }\end{array}$ & $\begin{array}{c}\mathrm{CL}+\mathrm{BG}, \\
\text { photoelectrons }\end{array}$ & $\begin{array}{c}\mathrm{CL}+\mathrm{BG}, \\
>200 \text { phel }\end{array}$ \\
\hline 60, proton & 18 & 20 & 18 \\
100, proton & 13 & 16 & 16 \\
\hline $30, \gamma$ & 5 & 10 & 9 \\
$50, \gamma$ & 2 & 9 & 8 \\
\hline
\end{tabular}

(e.g., 200 photoelectrons) for each fast detector somewhat reduces the uncertainties.

\subsection{CL spatial-angular distribution processing results}

The method for processing of CL angular images uses the spot shape parameters that provide maximum separation of showers initiated by primaries of different types. We calculate the integrals $S_{i}, i=1,2,3,4$ over rectangular segments of the FOV in the vicinity of the CL spot and use their ratios $r_{i j}=S_{i} / S_{j}$ as features characterizing the events. Maps for $0.75^{\circ} \times 0.75^{\circ}$ pixel size are used because this resolution turned out to be the most effective in preliminary studies [1]. Figure 2 gives the geometry of the image processing.

Optimum selection criteria have been found for p-N and $\mathrm{N}-\mathrm{Fe}$ pairs at 1 and $10 \mathrm{PeV}$ for different core distances $R$. Generally, optimum $r_{i j}$ depends on the pair, $E_{0}$ and $R$. In each case the borders between criterion class distributions are adjusted so that misclassification errors (e.g., $P\{p \rightarrow N\}$ and $P\{N \rightarrow p\}$ ) are equal. Thus, Table 4 shows only one value. Errors for pure CL signal are slightly lower than for CL+BG. Analysis shows that integration intervals along the spot long axis, corresponding to optimum $r_{i j}$, mostly depend on $R$ and a universal feature $r=r(R)$ can be defined behaving almost optimally. Figure 3 presents integration limits for the universal feature as functions of $R$.

The same approach to CL images processing is capable of suppressing at least $99 \%$ of nuclear background in 30-100 TeV $\gamma$-ray detection. In this case borders between $\gamma$ and proton classes are placed to leave only one proton in the $\gamma$ domain. Best results for $\mathrm{CL}+\mathrm{BG}$ turned into photoelectrons are shown in Table 5.

\section{Charged particle detector network}

We consider a fragment of charged particle detector network of $5 \times 5$ detectors $1 \mathrm{~m} \times 1 \mathrm{~m}$ each in order to

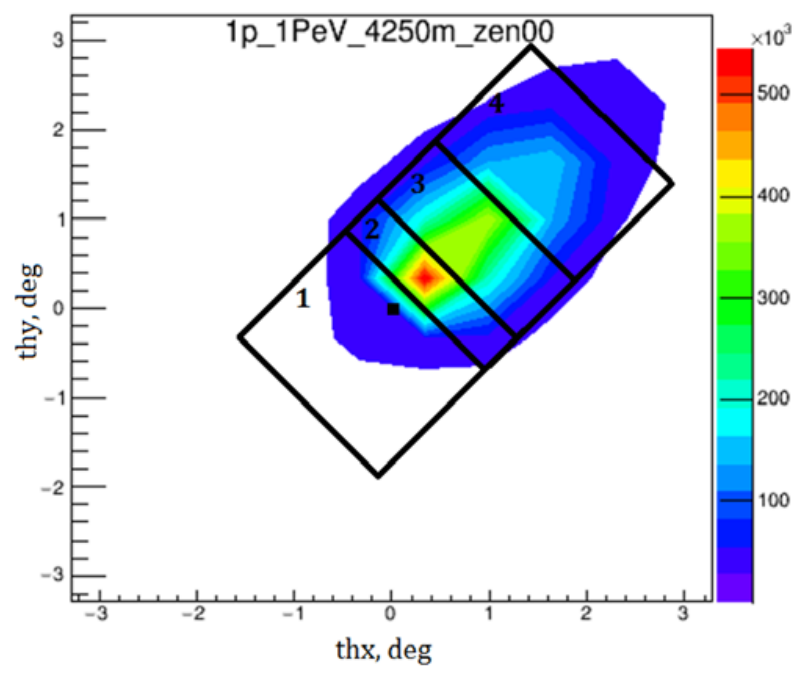

Figure 2. Shower image and rectangular areas of integration aligned along the spot long axis. Black square in the center of FOV marks the shower arrival direction. CL spot is sliced by rectangles 1,2,3,4 across its long axis. A stripe formed by the rectangles is $5^{\circ}$ wide. The outer side of rectangle 1 is placed at $-1.5^{\circ}$ with respect to the arrival direction. The image is integrated within the rectangles, integrals $S_{i}$ form ratios $r_{i j}$. The widths of the rectangles are varied so that to find $r_{i j}$ giving the best separation of images produced by different primary particles.

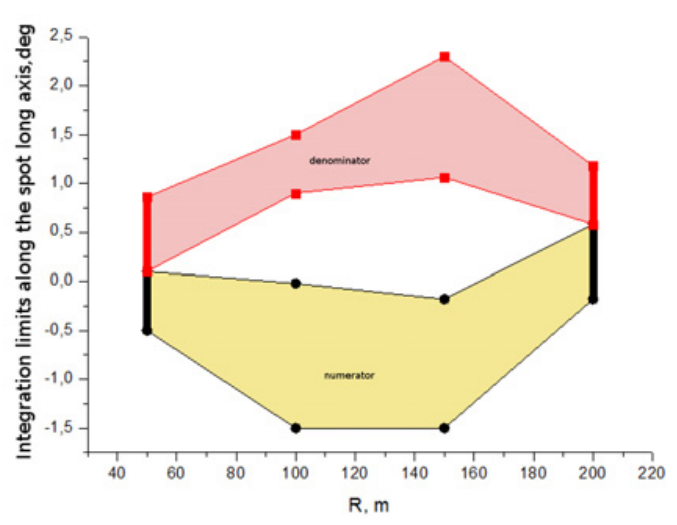

Figure 3. Integration limits for universal feature $r$ vs. $R$. Lower area: numerator integral. Upper area: denominator integral.

optimize the spacing (grid step) and find the lower limits of uncertainties in the core location, arrival direction and primary energy estimation. Two versions of particle spatial-temporal distribution processing are considered:

a) according to CORSIKA particle data, the number of charged particles and the moment of particle front arrival in each detector are defined; using 
Table 4. Misclassification probabilities for $\mathrm{p}-\mathrm{N}$ and N-Fe pairs, $E_{0}=1,10 \mathrm{PeV}$. All samples contain 240 images.

\begin{tabular}{l|l|l|llll|llll}
\hline \multirow{2}{*}{ signal } & \multirow{2}{*}{ criterion } & $E_{0}$ & \multicolumn{4}{|c|}{$1 \mathrm{PeV}$} & \multicolumn{4}{c}{$10 \mathrm{PeV}$} \\
\cline { 3 - 11 } & & $R, \mathrm{~m}$ & 50 & 100 & 150 & 200 & 50 & 100 & 150 & 200 \\
\hline CL, & \multirow{2}{*}{ photons } & $\mathrm{p}-\mathrm{N}$ & 0.05 & 0.10 & 0.15 & 0.17 & 0.09 & 0.13 & 0.17 & 0.21 \\
\hline CL+BG, & \multirow{2}{*}{ optimum } & $\mathrm{N}-\mathrm{Fe}$ & 0.05 & 0.10 & 0.15 & 0.15 & 0.004 & 0.04 & 0.09 & 0.12 \\
\hline photoelectrons & optimum & $\mathrm{p}-\mathrm{N}$ & 0.05 & 0.11 & 0.15 & 0.17 & 0.09 & 0.13 & 0.17 & 0.20 \\
& $\mathrm{~N}-\mathrm{Fe}$ & 0.05 & 0.10 & 0.15 & 0.16 & 0.01 & 0.04 & 0.09 & 0.13 \\
\hline photons & \multirow{2}{*}{ universal } & $\mathrm{p}-\mathrm{N}$ & 0.06 & 0.10 & 0.16 & 0.18 & 0.09 & 0.13 & 0.18 & 0.21 \\
& $\mathrm{~N}-\mathrm{Fe}$ & 0.05 & 0.11 & 0.15 & 0.16 & 0.004 & 0.05 & 0.10 & 0.12 \\
\hline CL+BG, & & $\mathrm{p}-\mathrm{N}$ & 0.05 & 0.11 & 0.15 & 0.17 & 0.09 & 0.13 & 0.17 & 0.20 \\
photoelectrons & \multirow{2}{*}{ universal } & $\mathrm{N}-\mathrm{Fe}$ & 0.05 & 0.11 & 0.17 & 0.16 & 0.01 & 0.05 & 0.10 & 0.13 \\
\hline
\end{tabular}

Table 5. Probabilities $P\{p \rightarrow \gamma\} / P\{\gamma \rightarrow p\}$. All samples contain 400 images.

\begin{tabular}{l|l|l}
\hline Pair & $50 \mathrm{~m}$ & $100 \mathrm{~m}$ \\
\hline $30 \mathrm{TeV} \gamma-60 \mathrm{TeV} \mathrm{p}$ & $0.0025 / 0.345$ & $0.0025 / 0.5125$ \\
$50 \mathrm{TeV} \gamma-100 \mathrm{TeV} \mathrm{p}$ & $0.0025 / 0.255$ & $0.0025 / 0.545$ \\
\hline
\end{tabular}

these measured data the core location and arrival direction are estimated as well as particle lateral distribution function which is then integrated to give a $E_{0}$ estimate;

b) all CORSIKA particles within a detector are tracked through its body ( $2 \mathrm{~mm}$ of $\mathrm{Al}+2 \mathrm{~cm}$ of polystyrene), by the deposited energies and centers of mass of ionization pulses the same event parameters as in a) are estimated.

$10 \mathrm{PeV}$ proton showers are used. In case a) 60 events are considered, in case b) 10 events. In both cases each event is used many (1600) times. Three grid steps are tried: 10, 15 and $20 \mathrm{~m}$. The shower axis is kept within the detector network fragment.

Step $15 \mathrm{~m}$ shows the best overall results in both cases, uncertainties are very similar: core location $\sim 1 \mathrm{~m}^{2}$, arrival direction $\sim 0.18^{\circ}$, primary energy $\sim 12 \%$. $E_{0}$ is estimated by the integral of particle LD within a circle of $100 \mathrm{~m}$ radius in case a) or of ionization LD within the same radius as in case b).

\section{Conclusions}

1. The proposed optical part of Pamir-XXI is capable of achieving the required accuracies in core location $(\sim 1 \mathrm{~m})$, arrival direction (better than $\left.0.1^{\circ}\right)$ and primary energy $(\lesssim 15 \%)$ within the target primary energy range $30 \mathrm{TeV}-100 \mathrm{PeV}$. Using this detector array it is also possible to divide all primary nuclei into three groups $(1-100 \mathrm{PeV})$ and reject not less than $99 \%$ of nuclear background events while selecting $\gamma$-events $(30-100 \mathrm{TeV})$.

2. The charged particle network can estimate the core location within $\sim 1 \mathrm{~m}$ accuracy, arrival direction with uncertainty $\sim 0.2^{\circ}$ and primary energy with uncertainty better than $15 \%$. A preferable grid step is $15 \mathrm{~m}$.

3. The question of whether and how this is possible to estimate the primary mass using charged particle detector data is still open. Probably, it is possible with muon trackers and/or central calorimeter with high spatial resolution.

\section{References}

[1] A. S. Borisov and V. I. Galkin, Journal of Physics: Conference Series, 409, No.1, 012089 (2013)

[2] D. Heck and T. Pierog Extensive Air Shower Simulation with CORSIKA: A User's Guide, (Karlsruher Institut fur Technologie, 2011) 